\title{
SCIENCE, TECHNOLOGIES AND INNOVATION IN GEORGIA IN THE DIMENSION OF GLOBAL EVALUATIONS: TRENDS AND CHALLENGES
}

\author{
ELENE PITSKHELAURI \\ PhD Student \\ Ivane Javakhishvili Tbilisi State University, Georgia \\ elene.pitskhelauri.12@gmail.com
}

\begin{abstract}
The paper explores the development of science, technology and innovation in Georgia over the last decade, reveals main tendencies, strengths and weaknesses. In a modern world, complex indexes are effectively utilized in order to evaluate the development of ST\&I, perform a comparative analysis and ranking countries. This itself supports the definition of economic strategies in the countries for increasing the productivity and long-term development. The goal of study was to evaluate the tendencies of ST\&I in Georgia for the time period of 2009-2019. A comparative analysis of progress has been carried out using Global Index of Innovation (GII) and the Global Competitiveness Index. According to the Global Innovation Index, the position of Georgia has significantly improved during the last 10 years and especially in the past 2 years. In comparison to 2015, Georgia improved its position in world ranking by 25 positions and by 50 positions - in comparison to 2009. In the report 2019 of Georgiass Innovation and Technology Agency, it is highlighted that Georgia is innovatively better in comparison to its GDP (Cornell University \& INSEAD \& WIPO, 2019: 20). Georgia has the highest ranking in the following pillars of the index: institutional structure ( score $-74,3$ and ranking -26 ) and market sophistication (score -62.1 and ranking -15). In other pillars the country ranking range varies between 58-72 and score between 22.5-44.7. During 2011-2019, among all pillars of the index the following areas reached the highest scores in 2019: institutions, infrastructure and market sophistication. During the last decade all pillars of the index are progressing except knolwedge and technology outputs. In these the position and rating of Georgia has even become worse; in comparison to 2011 the ranking of Georgia in this group dropped and went down 25 positions. In 2019 Georgia's Innovation and Technology Agency defined the strongest 10 indicators out of 98 indicators of sub-pillars; among these there are categories where Georgia has not progressed during 2011-2019. These are: Human resources and research, knowledge and technology and creative outputs. However, it has to be mentioned that the following sub-pillars are considered as the weakness of Georgia and consequently have a lower rating: ecological sustainability (score 91), knowledge diffusion (score 86), knowledge workers (score 81), trade, competition and market scale (score 79), knowledge absorbation (78), reasearch and development (75) (Cornell University, INSEAD, and WIPO, 2019: 258). According to Global Competetiveness Index and similar to Global Innovation Index the position of Georgia during 2009-2018 has significantly improved. In comparison to 2009, Georgia went up 24 positions. The data show that the group of innovation capability is the greatest challenge for achieving a high score on a global scale. Despite the fact that GII in Georgia is characterized by improving tendency during 2009-2019, the analysis of indexes confirms that the hindering factors for achieving high score on a global scale and becoming a competetive country for Georgia are: knowledge and technology, research and development, creativity and innovation capabilities; this reconfirms that the development of science, technology and innovation remains one of the major challenges for Georgia.
\end{abstract}

KEYWORDS: SCIENCE, TECHNOLOGY, INNOVATION, GLOBALIZATION, GLOBAL INDEXES, STATISTICAL TRENDS.

For citation: Pitskhelauri E. (2020). Science, Technologies and Innovation in Georgia in the Dimension of Global Evaluations: Trends and Challenges. Globalization and Business, №9, pp. 156-162 (In Georgian).

https://doi.org/10.35945/gb.2020.09.019 


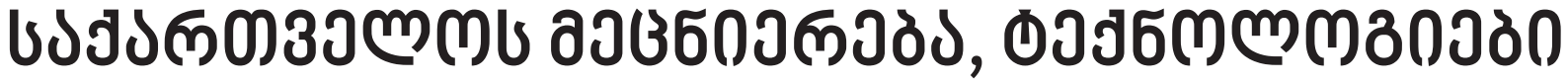

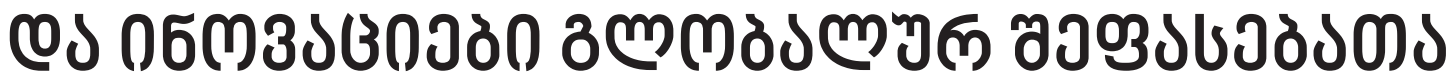

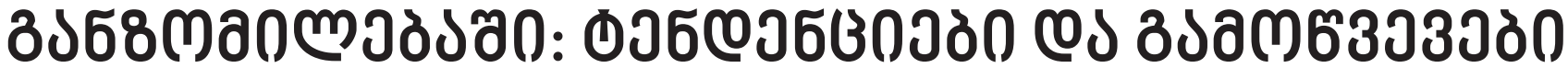

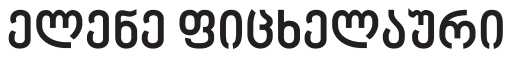

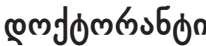

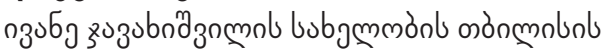

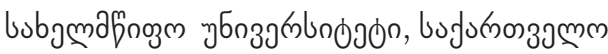

elene.pitskhelauri.12@gmail.com

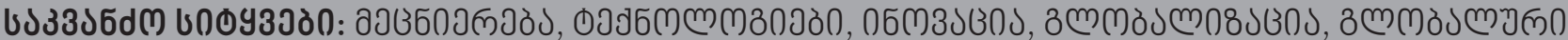

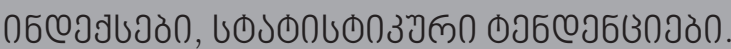

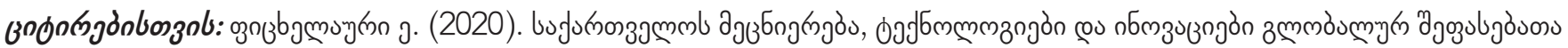

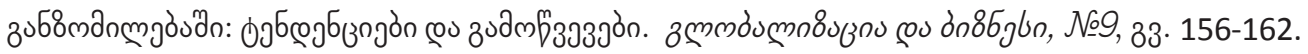

https://doi.org/10.35945/gb.2020.09.019

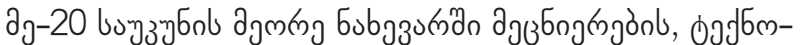

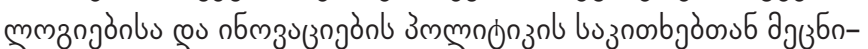

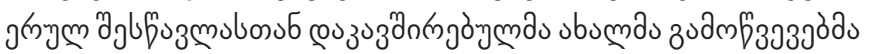

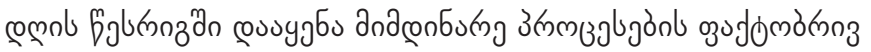
dubumusog cougyd

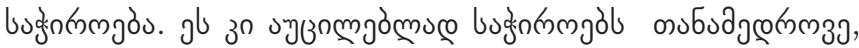

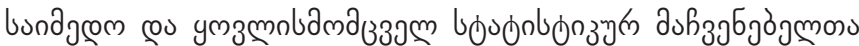

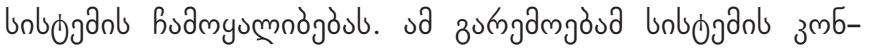

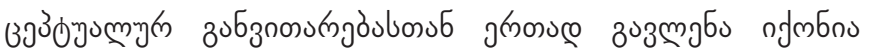

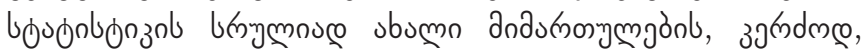

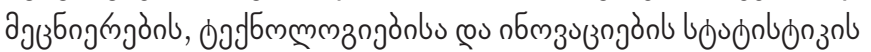
hodmyumnojjö80 (Inzelt, 2008: 9).

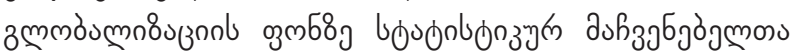

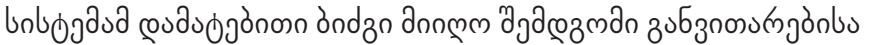

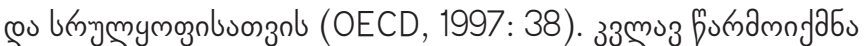

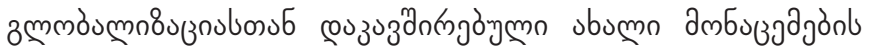

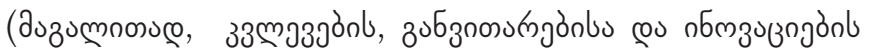

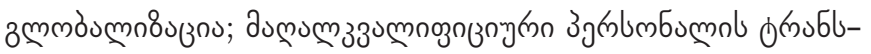

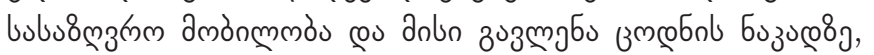

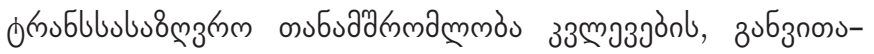

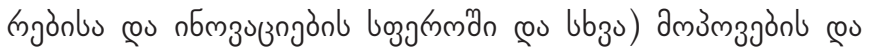

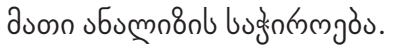

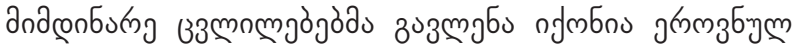

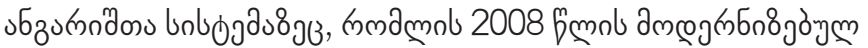

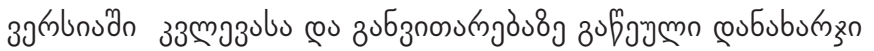

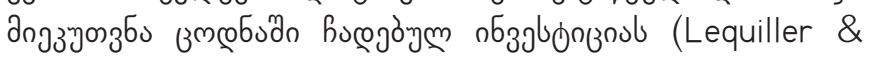
Blades, 2014: 5).

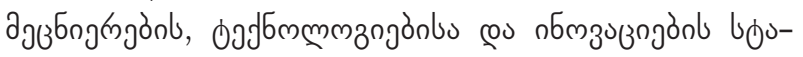

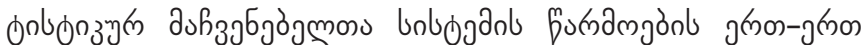

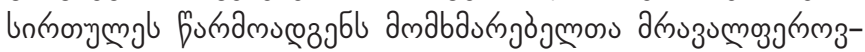

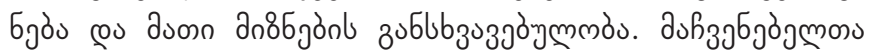

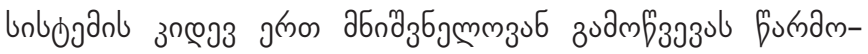

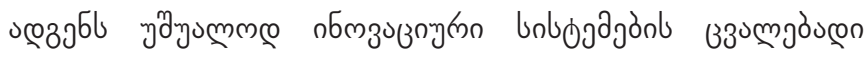

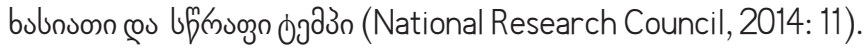

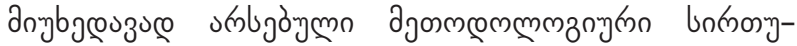

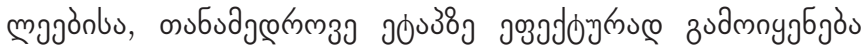

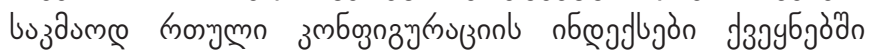

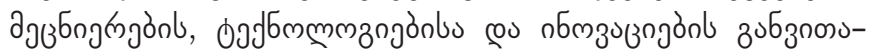

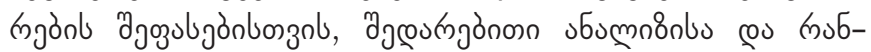

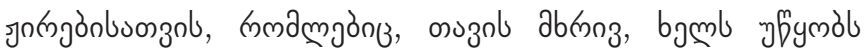

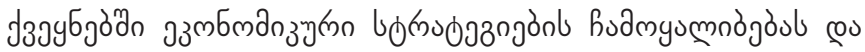

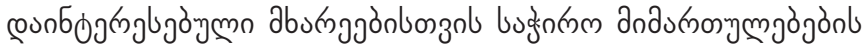

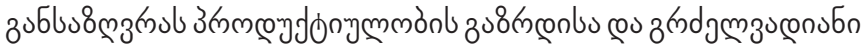
зuбznosumgonnbuonzol.

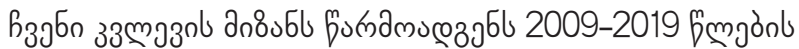

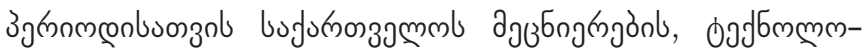

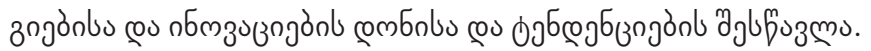

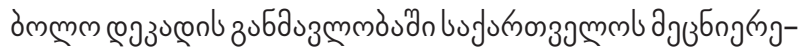

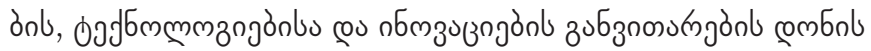

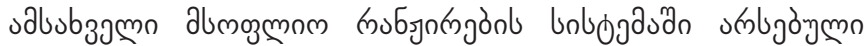

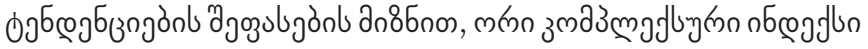

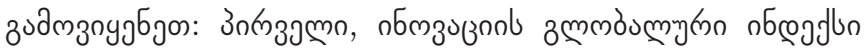

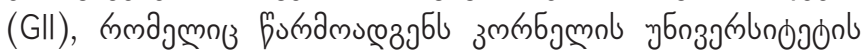

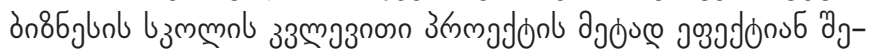

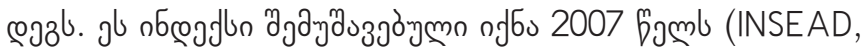

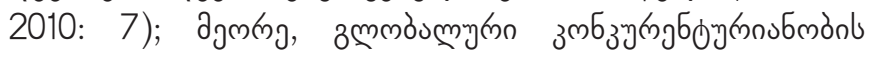




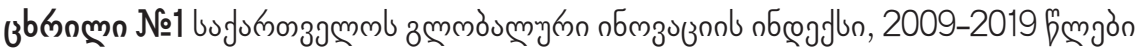

\begin{tabular}{|c|c|c|c|c|c|c|c|c|c|c|c|}
\hline 6эmo & 2009 & 2010 & 2011 & 2012 & 2013 & 2014 & 2015 & 2016 & 2017 & 2018 & 2019 \\
\hline 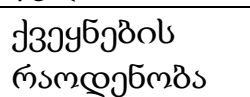 & 130 & 132 & 125 & 141 & 142 & 143 & 141 & 128 & 127 & 126 & 129 \\
\hline 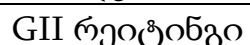 & 98 & 84 & 73 & 71 & 59 & 71 & 73 & 64 & 68 & 59 & 48 \\
\hline
\end{tabular}

fyurnm: Cornell University, INSEAD, and WIPO, 2019

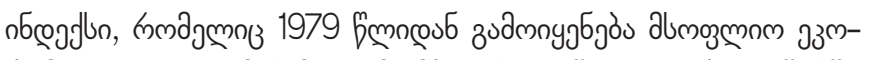

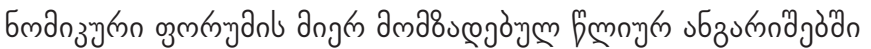
(Schwab, 2018: 1).

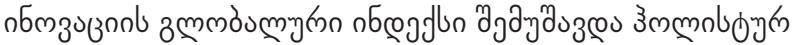

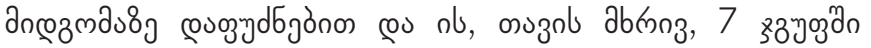

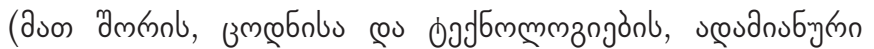

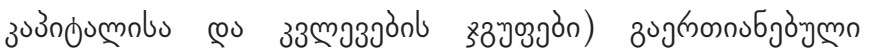

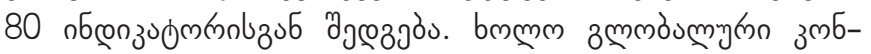

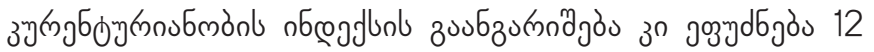

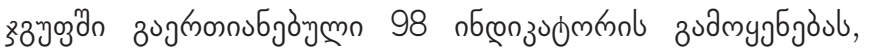

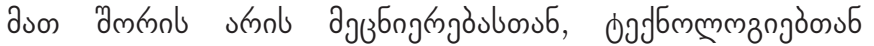

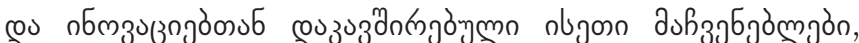

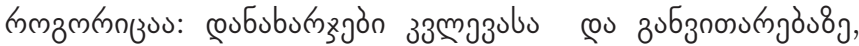

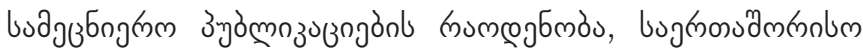

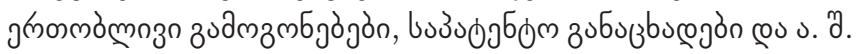

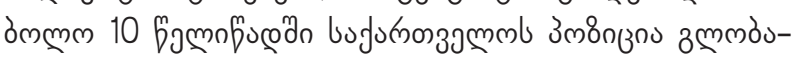

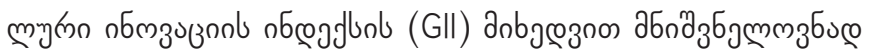
s

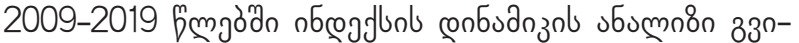

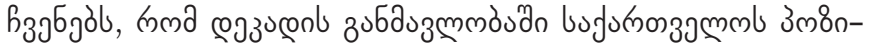

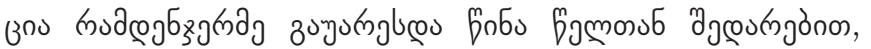

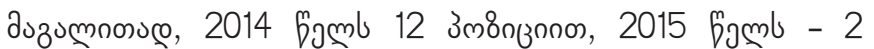

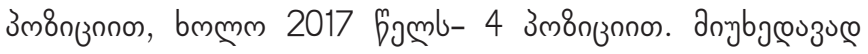

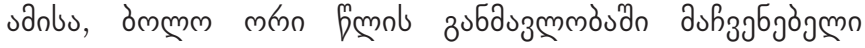

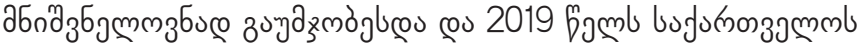

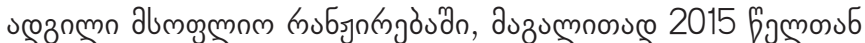

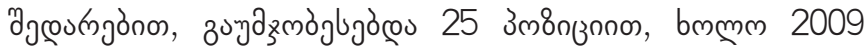

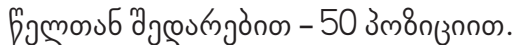

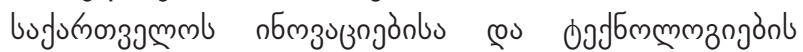

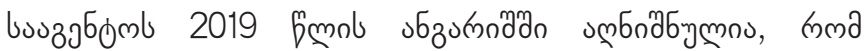

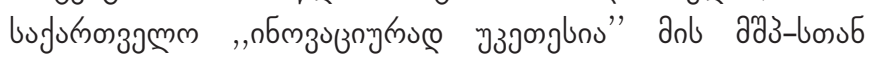

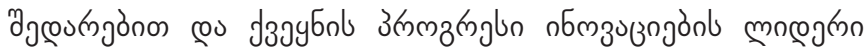

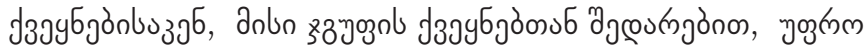
uffromos (Cornell University \& INSEAD \& WIPO, 2019: 20).

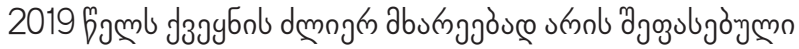

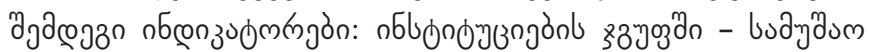

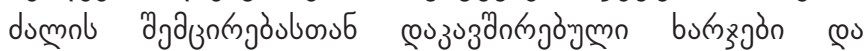

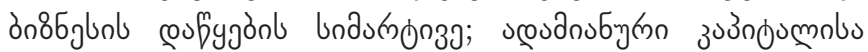

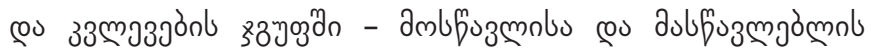

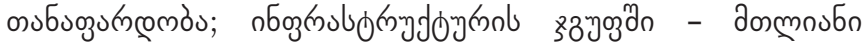

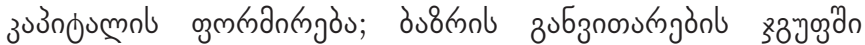

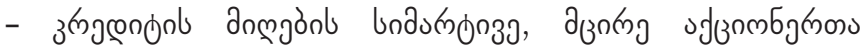

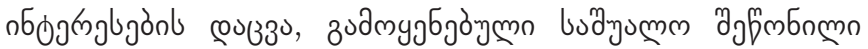

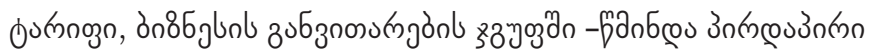

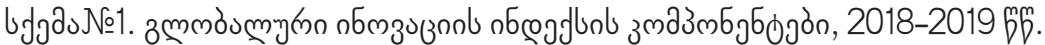

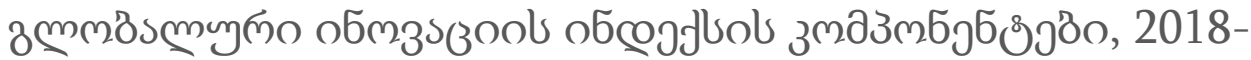
2019 6ேmjס̊०
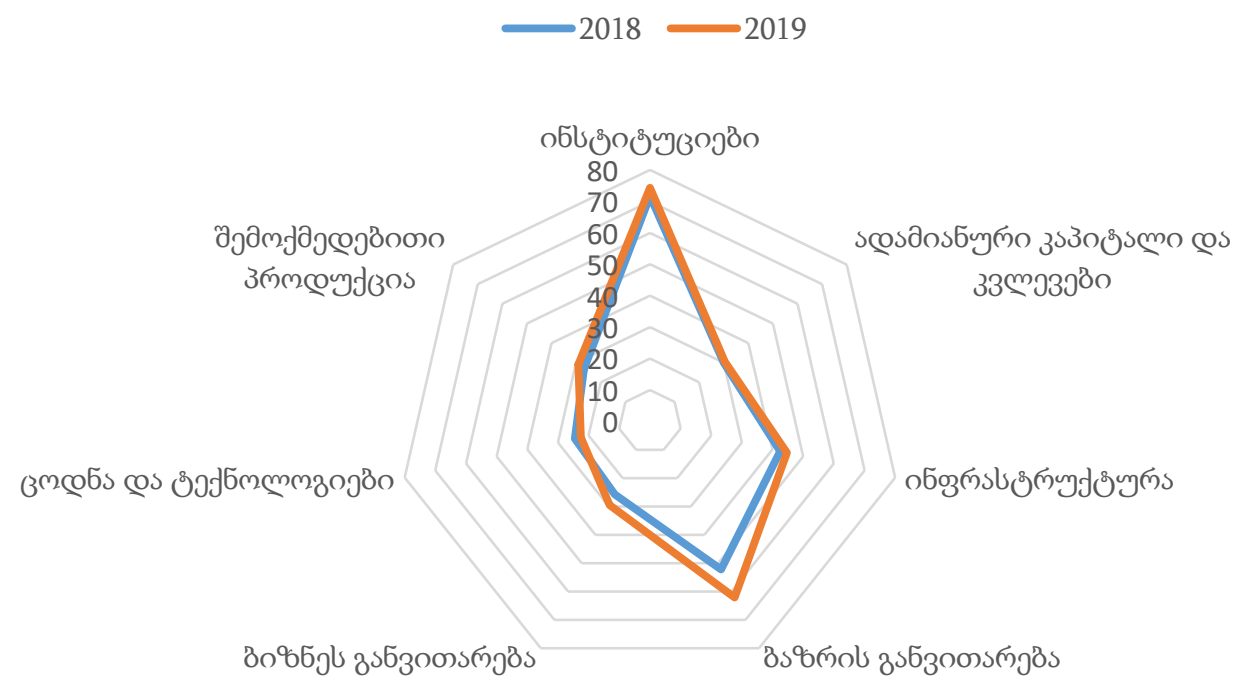


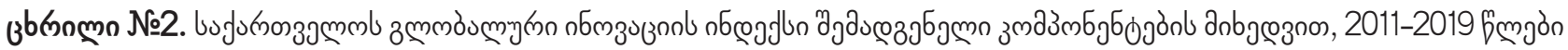

\begin{tabular}{|c|c|c|c|c|c|c|c|c|c|c|c|}
\hline \multicolumn{2}{|c|}{ 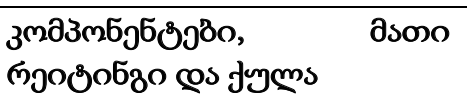 } & 2011 & 2012 & 2013 & 2014 & 2015 & 2016 & 2017 & 201 & & 2019 \\
\hline \multicolumn{2}{|l|}{ GII ๓ூ๐லீం5ூం } & 73 & 71 & 59 & 71 & 73 & 64 & 68 & 5 & & 48 \\
\hline \multirow{2}{*}{\multicolumn{2}{|c|}{ 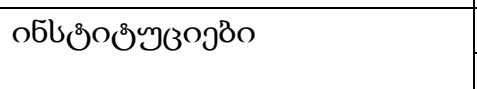 }} & 45 & 51 & 47 & 46 & 51 & 44 & 47 & 3 & & 36 \\
\hline & & 72,4 & 65,2 & 69,4 & 69,7 & 68,2 & 69,2 & 68,6 & 71 & & $(74,3)$ \\
\hline \multirow{2}{*}{\multicolumn{2}{|c|}{ 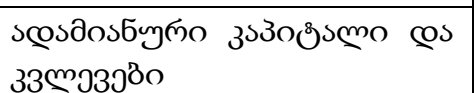 }} & 83 & 95 & 97 & 90 & 91 & 91 & 89 & 6 & & $63^{-6}$ \\
\hline & & $32,6)$ & 29,6 & 24,9 & 23,5 & 23,6 & 23,2 & 23,6 & 30 & & 30,5 \\
\hline \multirow{2}{*}{\multicolumn{2}{|c|}{ 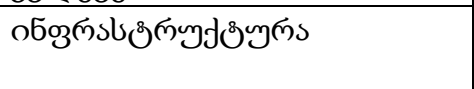 }} & '1+1's' & 87 & 72 & 82 & 79 & 69 & 74 & 7 & & 72 \\
\hline & & 20,2 & 29,4 & 31,2 & 33,3 & 36,6 & 41,7 & 43,8 & 42 & & 44,7 \\
\hline \multirow{2}{*}{\multicolumn{2}{|c|}{ 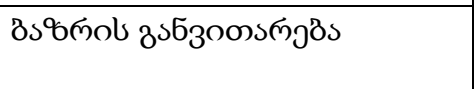 }} & 58 & 31 & 34 & 37 & 42 & 55 & 53 & 3 & & 155 \\
\hline & & 41,1 & 50,3 & 54,5 & 55,2 & 52,8 & 44,3 & 49,2 & 52 & & $(62,1)$ \\
\hline \multirow{2}{*}{\multicolumn{2}{|c|}{ 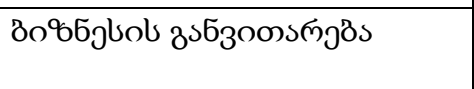 }} & 103 & -96 & 95 & 119 & 105 & 90 & 101 & 9 & & $7 \theta^{-1}$ \\
\hline & & 26,4 & $(34,0)$ & 28,0 & 23,9 & 28,0 & 26,5 & 25,6 & 25 & & 29,5 \\
\hline \multirow{2}{*}{\multicolumn{2}{|c|}{ 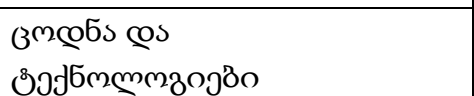 }} & 37 & 57 & 63 & 58 & 67 & 56 & 54 & 5 & & 62 \\
\hline & & $30,6)$ & 29,5 & 27,0 & 30,0 & 26,6 & 26,8 & 23,9 & 24 & & 22,5 \\
\hline \multirow{2}{*}{\multicolumn{2}{|c|}{ 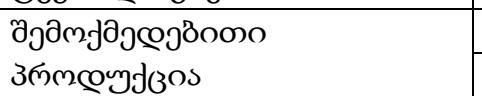 }} & Tr4t' & 105 & -95 & 99 & 99 & 76 & 69 & 7 & & 58 \\
\hline & & 19,8 & 24,2 & 32,0 & 25,9 & 25,0 & 26,6 & 29,3 & 26 & & 29,1 \\
\hline \begin{tabular}{l|l} 
6jmo & 2009
\end{tabular} & 2010 & 2011 & 2012 & 2013 & 2014 & 2015 & 2016 & 2017 & 2018 & 2019 & \\
\hline
\end{tabular}

fyurnm: Cornell University, INSEAD, and WIPO, 2019

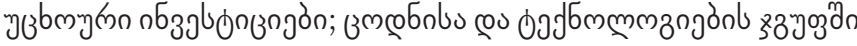

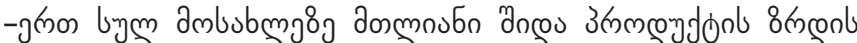

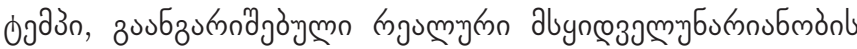

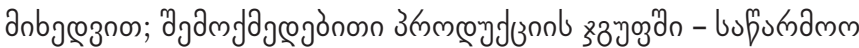

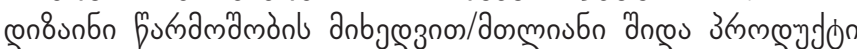

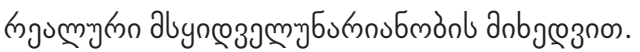

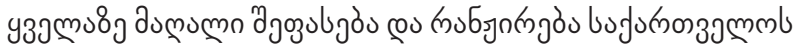

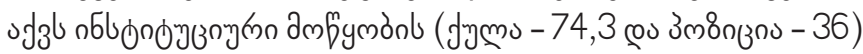

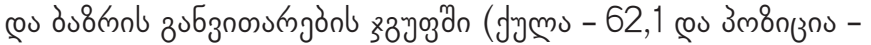

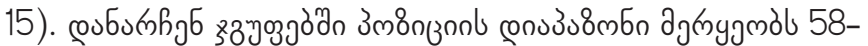

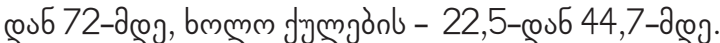

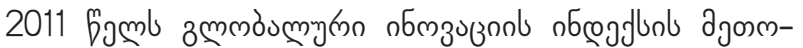

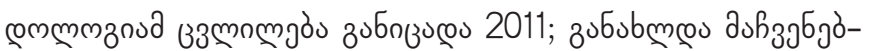

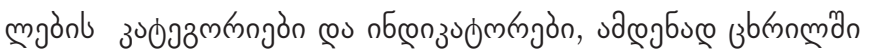

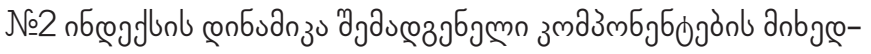

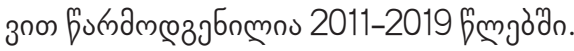

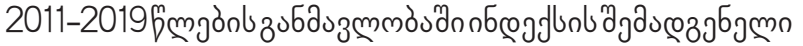

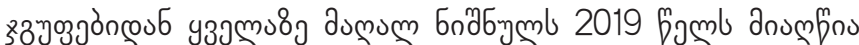

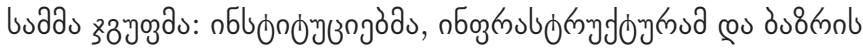

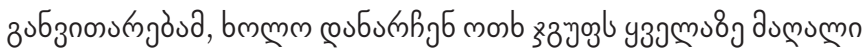

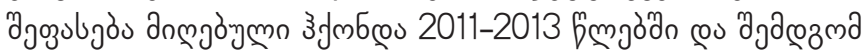
Бм

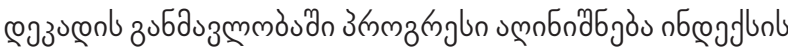

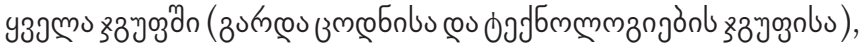

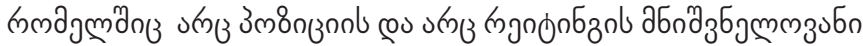

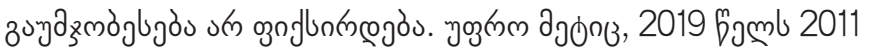

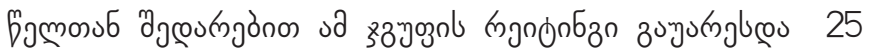

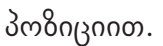

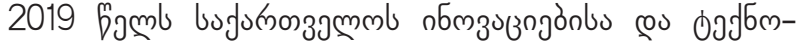

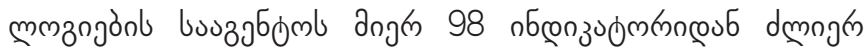

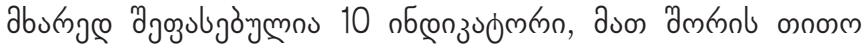

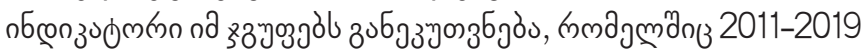

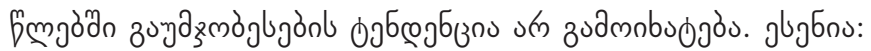

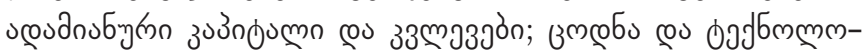

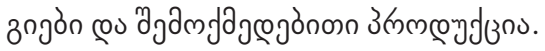

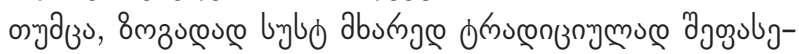

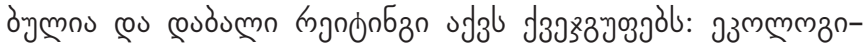

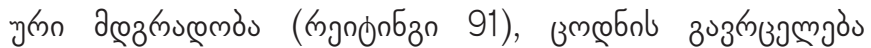

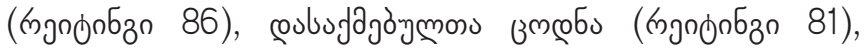

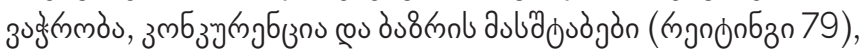

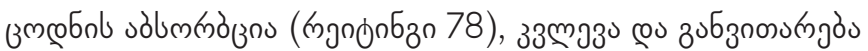
(mgnonfän 75) (Cornell University, INSEAD, and WIPO, 2019: 258).

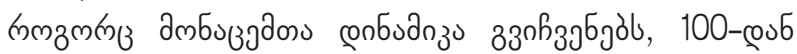

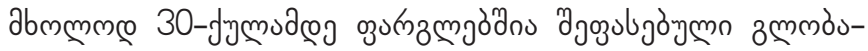

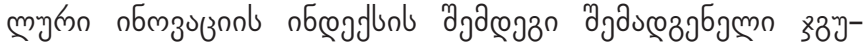

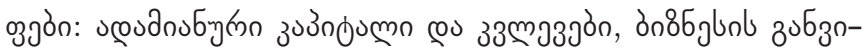

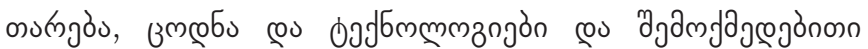

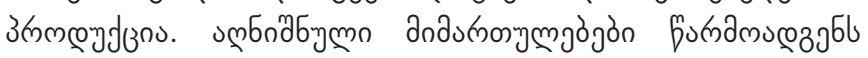

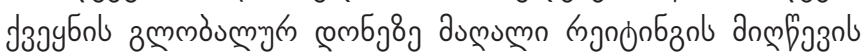

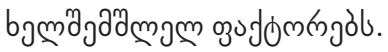

з змmöum

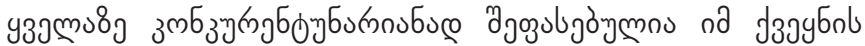

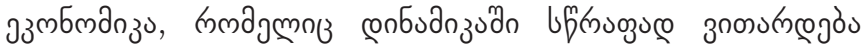
(World Economic Forum, 2019: 235).

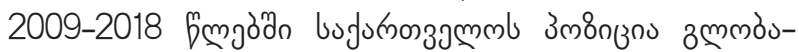




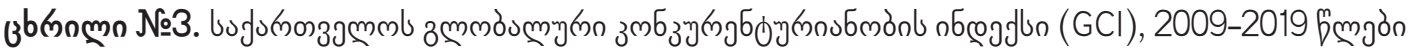

\begin{tabular}{|c|c|c|c|c|c|c|c|c|c|c|c|}
\hline 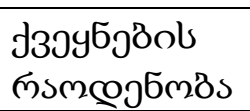 & 133 & 139 & 142 & 144 & 148 & 144 & 140 & 138 & 137 & 140 & 140 \\
\hline $\begin{array}{l}\text { GCI } \\
\text { ๓ృ๐ళீం5วం }\end{array}$ & 90 & 93 & 88 & 77 & 72 & 69 & 66 & 59 & 67 & 66 & $*$ \\
\hline
\end{tabular}

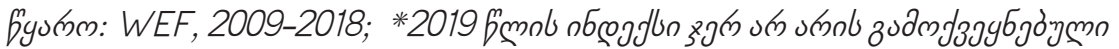

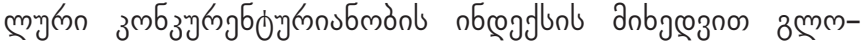

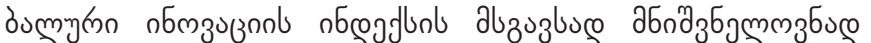

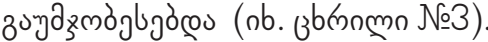

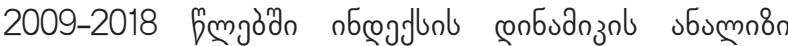

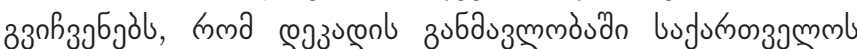
उm8n

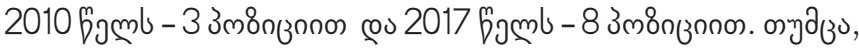

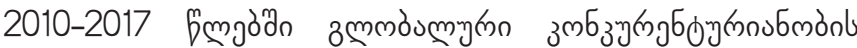

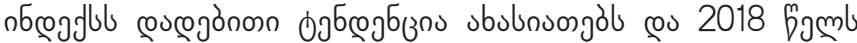

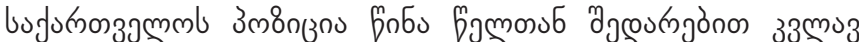

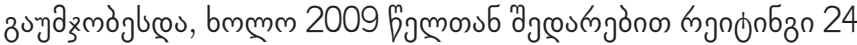

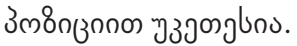

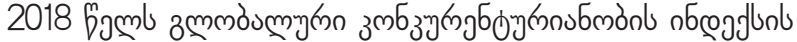

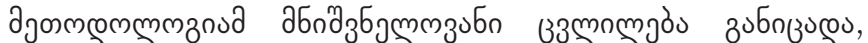

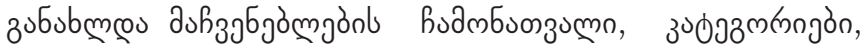

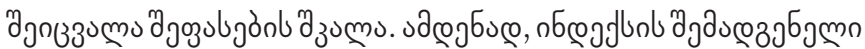

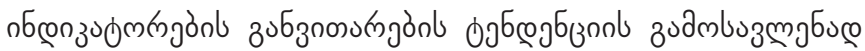

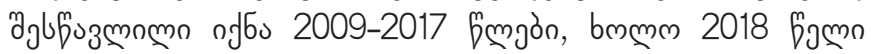

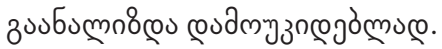

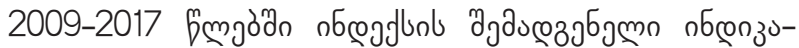

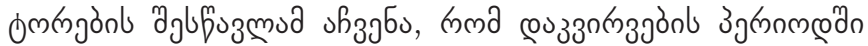

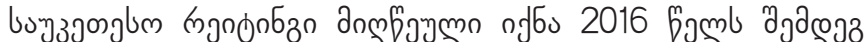

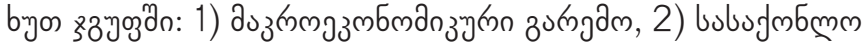

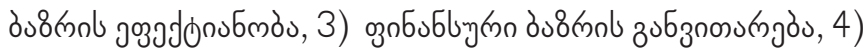

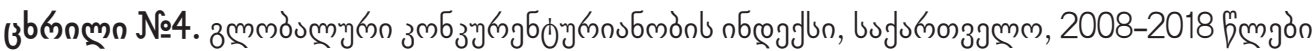

\begin{tabular}{|c|c|c|c|c|c|c|c|c|c|}
\hline 6כmo & 2009 & 2010 & 2011 & 2012 & 2013 & 2014 & 2015 & 2016 & 2017 \\
\hline 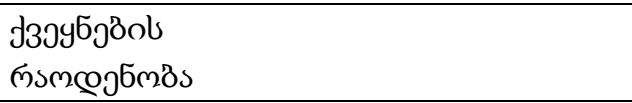 & 133 & 139 & 142 & 144 & 148 & 144 & 140 & 138 & 137 \\
\hline 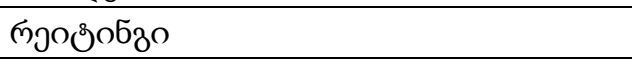 & 90 & 93 & 88 & 77 & 72 & 69 & 66 & 59 & 67 \\
\hline jyms & 3,8 & 3,9 & 4,0 & 4,1 & 4,2 & 4,2 & 4,2 & 4,3 & 4,3 \\
\hline \multicolumn{10}{|l|}{ 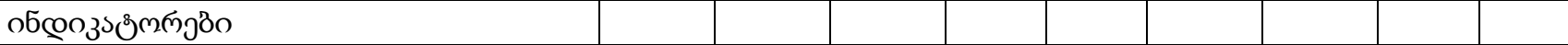 } \\
\hline 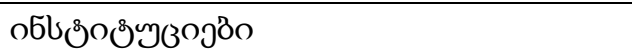 & 72 & 69 & 60 & 61 & 64 & 48 & 40 卉 & 43 & 50 \\
\hline 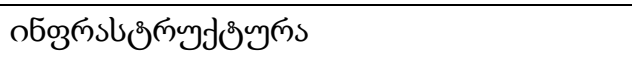 & 72 & 73 & 68 & $53+1$ & 56 & 59 & 61 & 65 & 69 \\
\hline 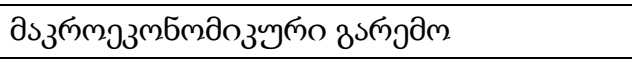 & 117 & 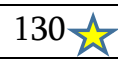 & $137 \sqrt{4}$ & 88 & 61 & 48 & 51 & 40 弐 & 48 \\
\hline 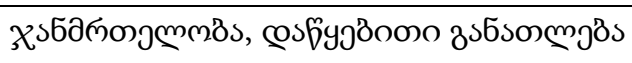 & 78 & 73 & 67 & 61 志 & 70 & 63 & 65 & 64 & 69 \\
\hline 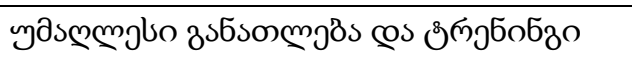 & 84 龸 & 90 & 88 & 93 & 92 & 92 & 87 & 89 & 87 \\
\hline 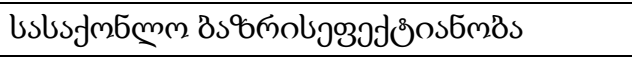 & 74 & 64 & 74 & 82 & 67 & 60 & 48 & 46 氠 & 50 \\
\hline 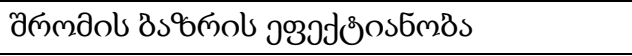 & 17 可 & 31 & 32 & 35 & 40 & 41 & 32 & 43 & 53 \\
\hline 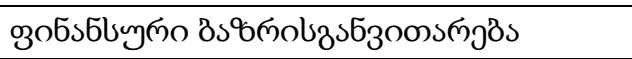 & 95 & 108 & 99 & 93 & 75 & 76 & 68 & 58 牙 & 63 \\
\hline 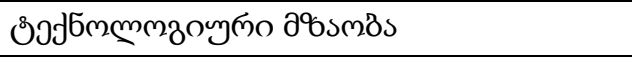 & 100 & 98 & 100 & 76 & 68 & 67 & 72 & 65 牙 & 70 \\
\hline 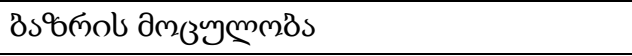 & 101 & 107 & 106 & 99 本 & 103 & 103 & $99 \star$ & 101 & 100 \\
\hline 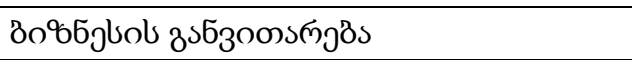 & 113 & 111 & 111 & 113 & 120 & 113 & 112 & 102 & 99 孛 \\
\hline обмзаззојво & 119 & 125 & 118 & 126 & 126 & $121\}$ & 123 క & 116 志 & $118 \underline{y}$ \\
\hline
\end{tabular}

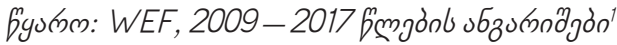

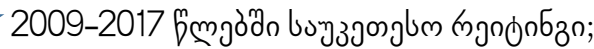

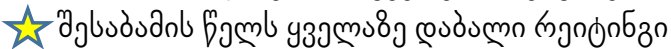

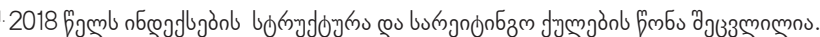




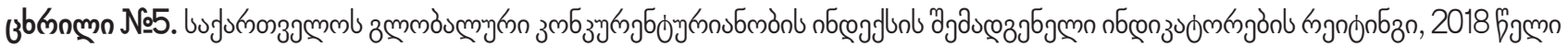

\begin{tabular}{|c|c|c|c|c|c|}
\hline \multicolumn{3}{|l|}{ GCI ๓довобабо } & \multicolumn{3}{|c|}{ 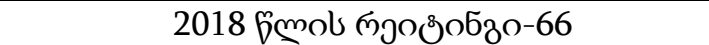 } \\
\hline 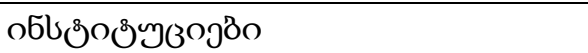 & 40 & +10 & 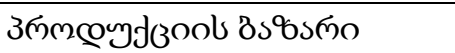 & 42 & +8 \\
\hline 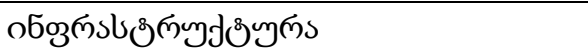 & 72 & -3 & 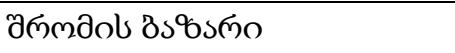 & 31 & +22 \\
\hline 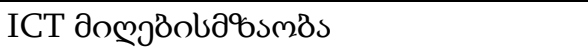 & 45 & +25 & 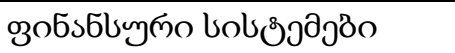 & 88 & -25 \\
\hline 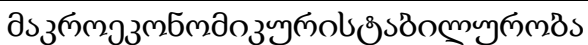 & 72 & -24 & 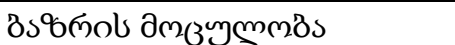 & 102 & -2 \\
\hline хзбаполмммдs & 80 & - & 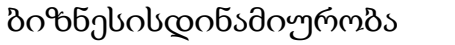 & 53 & +46 \\
\hline y5sলngoo & 45 & - & 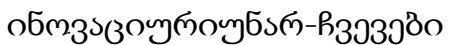 & 85 & - \\
\hline
\end{tabular}

fyumm: WEF, 2018

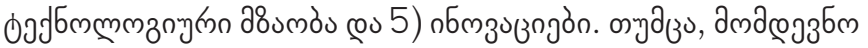

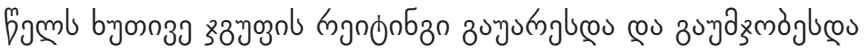

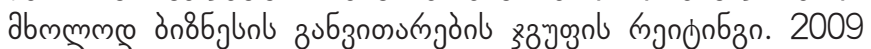

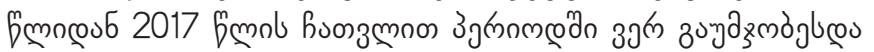

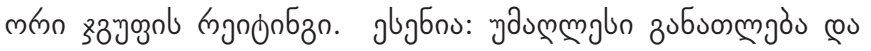

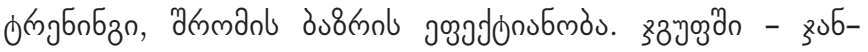

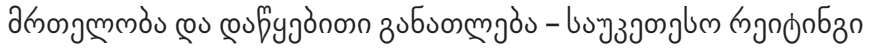

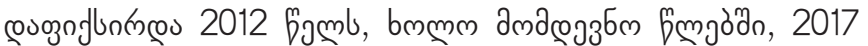

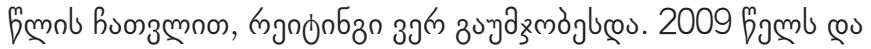

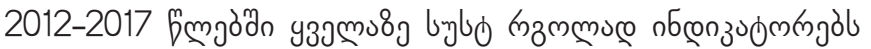

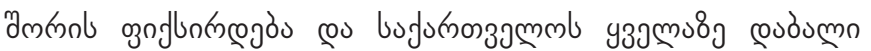

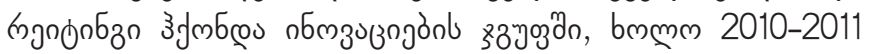

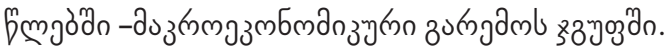

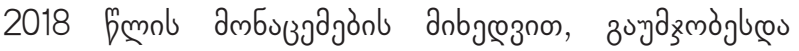

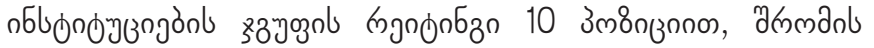

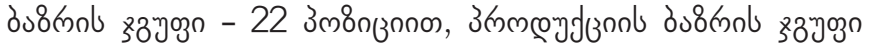

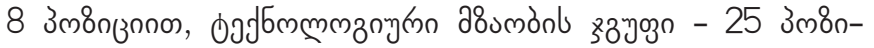

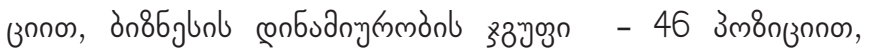

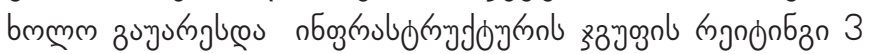

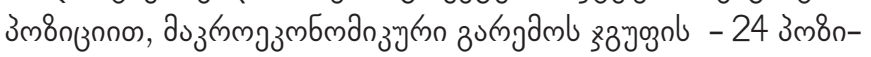

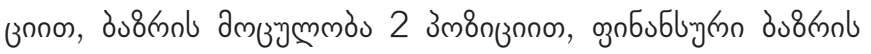

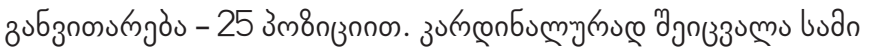

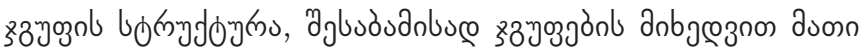

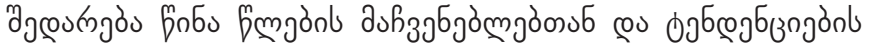

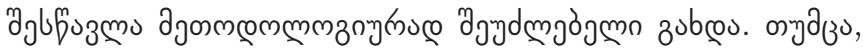

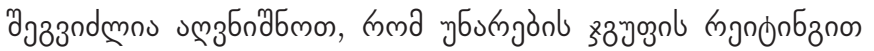

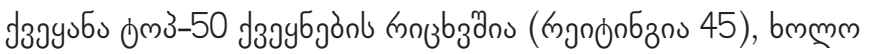

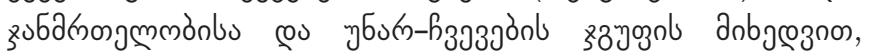

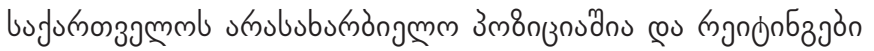

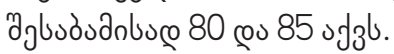

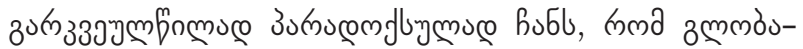

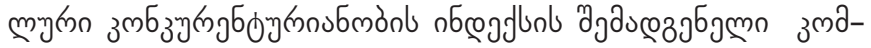

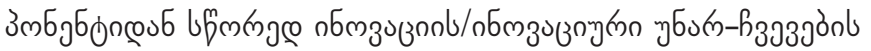

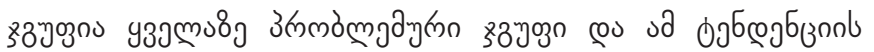

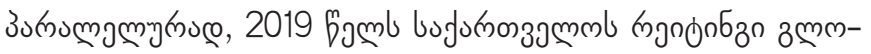

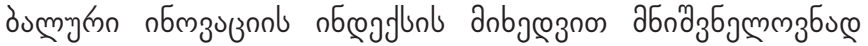
зuगdzmòglos.

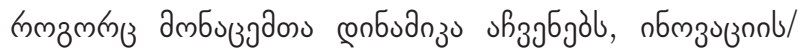

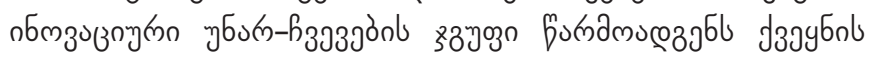

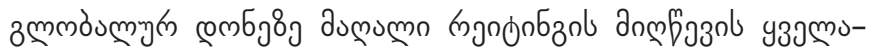

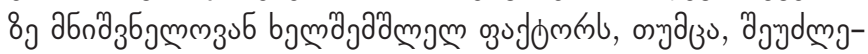

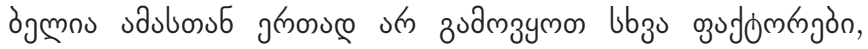

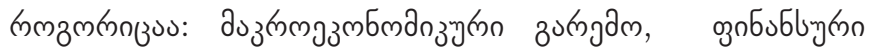

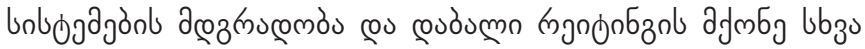

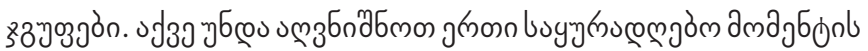

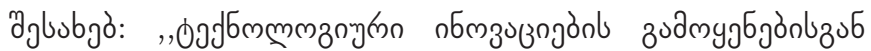

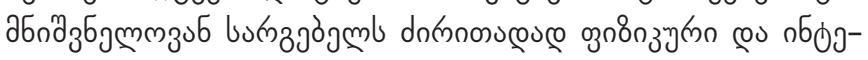

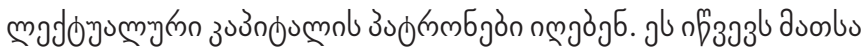

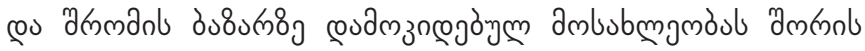

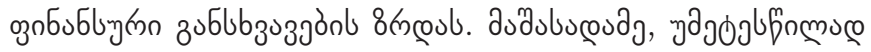

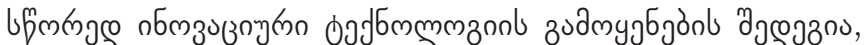

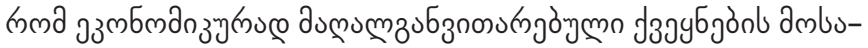

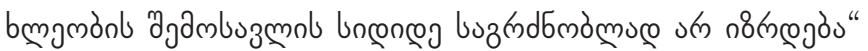
(Gelashvili, 2018: 71).

\section{esu336s}

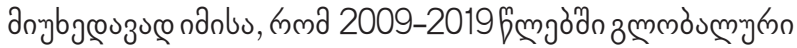

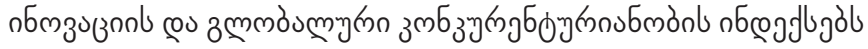

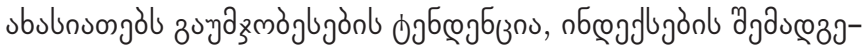

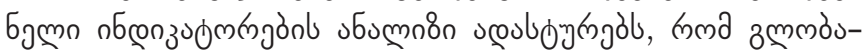

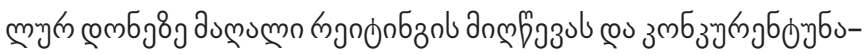

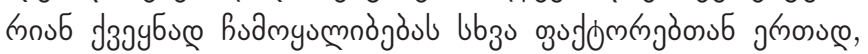

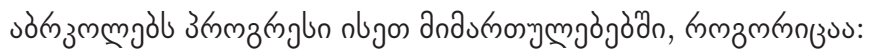

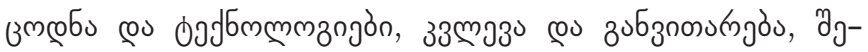

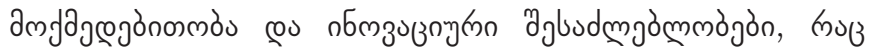

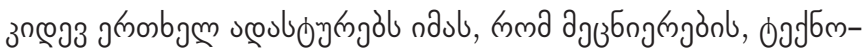

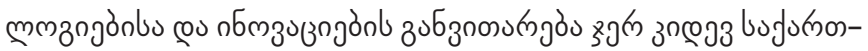

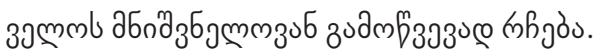




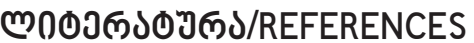

Cornell University, INSEAD, and WIPO (2019); The Global Innovation Index 2019: Creating Healthy Lives - The Future of Medical Innovation, Ithaca, Fontainebleau, and Geneva.

Gelashvili S. (2018). The Interconnection of the use of Human Physical Abilities and Global Technological Progress. Journal "Globalization \& Business”, №6, 2018, p. 67-72.

Inzelt, A .(2008). Emergence of new branches of statistics Science, technology and innovation statistics. In: Péter Kovács Katalin, Szép Tamás Katona. Challenges for Analysis of the Economy, the Businesses and Social Progress. Universitas Szeged Press.

Lequiller, F. and D. Blades (2014). Understanding National Accounts: Second Edition, OECD Publishing.

National Research Council (2014).Capturing Change in Science, Technology and Innovation: Improving Indicators to Inform Policy. Panel on Developing Science, Technology and Innovation Indicators for the Future. R.E. Litan, A.W. Wyckoff and K.H. Fealing, Editors. Committeeon National Statistics, Division of Behavioral and Social Sciences and Education.

Technology and Economic Policy. Division of Policy and Global Affairs. Washington, DC: The National Academies Press. OECD (1997). National Innovation Systems. OECD Publishing, Paris.

Schwab, K. (2019). The Global Competitiveness Report 2018. World Economic Forum. 